\title{
Covert Verb Reading Contributes to Signal Classification of Motor Imagery in BCI
}

\author{
Hong Zhang, Yaoru Sun*, Jie Li, Fang Wang, Zijian Wang
}

\begin{abstract}
Motor imagery is widely used in the brain-computer interface (BCI) systems that can help people actively control devices to directly communicate with the external world, but its training and performance effect is usually poor for normal people. To improve operators' BCI performances, here we proposed a novel paradigm, which combined the covert verb reading in the traditional motor imagery paradigm. In our proposed paradigm, participants were asked to covertly read the presented verbs during imagining right hand or foot movements referred by those verbs. EEG signals were recorded with both our proposed paradigm and the traditional paradigm. By the common spatial pattern (CSP) method, we respectively decomposed these signals into spatial patterns and extracted their features used in the following classification of support vector machine (SVM). Compared with the traditional paradigm, our proposed paradigm could generate clearer spatial patterns following a somatotopic distribution, which led to more distinguishable features and higher classification accuracies than those in the traditional paradigm. These results suggested that semantic processing of verbs can influence the brain activity of motor imagery and enhance the mu event-related desynchronisation (ERD). The combination of semantic processing with motor imagery is therefore a promising method for the improvement of operators' BCI performances.
\end{abstract}

Index Terms-BCI; covert verb reading; ERD; mu rhythm; motor imagery.

\section{INTRODUCTION}

$\mathrm{M}$ OTOR imagery can modify neural activity in motor cortices where the potential changes can be recorded by electroencephalogram (EEG) [1]. The activated areas are correlated with event-related desynchronisation (ERD) of mu and beta rhythm [2-6]. Corresponding to the type of motor imagery, the ERD was observed in the specific sensorimotor areas. For example, the imagery of left or right hand movement elicits ERD over the contralateral area, while the ERD of foot movement imagery localizes on the central area [7]. The different spatial distributions of ERD during imageries of

This work was supported by the Grants from the National Natural Science Foundation of China (61173116) and the Ministry of Science and Technology of China (2016YFC1306805).

*Corresponding author: Yaoru Sun, email: yaoru@tongji.edu.cn.

Hong Zhang is with Department of Computer Science and Technology, Tongji University, Shanghai, 201804, China; and with Department of Mathematics, Taiyuan Normal University, Taiyuan Shanxi, 030619, China.

*Yaoru Sun, Jie Li and Zijian Wang are with Department of Computer Science and Technology, Tongji University, Shanghai, 201804, China.

Fang Wang is with Department of Computer Science, Brunel University, Uxbridge UB8 3PH, United Kingdom. different movement enable us classify EEG signals according to the imagined movements and generate corresponding commands [4, 8]. Therefore, the EEG signals based on motor imagery are widely used in the brain-computer interface $(\mathrm{BCI})$ systems. By imagery of left hand, right hand or foot movement respectively, a three dimensional command can be generated to control the BCI system devices $[9,10]$, such as video games [11], wheelchair or virtual car [12, 13].

Successful application of motor imagery based BCI depends on the high accuracy of EEG signal classification. In recent years, more and more data analysis methods have been proposed for EEG signal processing to improve the classification performances [14-16], but users still need time-consuming practices for training before successfully operating the BCI devices. Moreover, the complexity of algorithms also limits the generalization of BCI systems. Hence, how to improve operators' performance, especially in the convenient and user-friendly BCI systems based on motor imagery, is still a hot issue. As we all know, the performance of a BCI system relies not only on the system models but also on users' brain activity patterns. Previous studies showed that feedback training is an effective method for participants to modulate the brain activity [17-21]. However, the performance of feedback-based BCI systems largely depends on the initial models constructed to provide the feedback. These initial models are calibrated with the data recorded at the beginning of the training sessions. The calibration data may not contain sufficient discriminability since participants are unfamiliar with the systems during the initial sessions. Therefore, the inaccurate feedback may be provided to the participants and frustrate them [22] and even produce an inhibitory effect on their EEG controls. There have also been other paradigms proposed to improve participants' BCI performance. For example, Li and Zhang [23] attributed the decreased BCI performance to incorrect responses to the cues. They proposed an active training paradigm, in which participants were required to reconfirm trial labels after the imagery. This paradigm overcame the imagery mistakes that did not match with the given cues, and achieved better performance than the traditional paradigm. Nevertheless, the active training paradigm needs pressing keys to reconfirm the labels and cannot work well for users with motor disability of hands, especially those with limb or general paralysis.

Embodied cognition demonstrates that language comprehension is closely linked to motor perception. Using event-related fMRI, Hauk et al. [24] showed that passively 
reading action words referring to hand, foot or mouth actions could activate relevant motor areas similar to performing those corresponding actions. Recent studies also found a specific somatotopic motor representation of different body parts within the premotor cortex when participants processed hand-, footand mouth-related verbs [25]. These results indicated an interaction between language and motor processing. Furthermore, there were some researches demonstrating that processing action words can significantly influence the motor responses [26-31]. It has been shown that motor-related word generation can facilitate finger-tapping, suggesting that the semantic-motor representation has an effect on motor production [30]. This effect was attributed to the co-activated motor cortex by processing of action words and motor actions [28]. Motor imagery is also suggested to activate the motor cortex [32]. Imagery of hand, foot or mouth movements can elicit brain activity in the corresponding motor cortex [33,34]. Therefore, we hypothesized that processing of action words or verbs could influence the brain activity of motor imagery and would significantly improve the EEG signal classification.

In this study, we proposed a novel paradigm, in which the cue stimuli involved not only an arrow labeling the action imagery but also a verb. Participants were asked to read the presented verb covertly during motor imagery of the movement referred by that verb. To compare our paradigm with the traditional paradigm, we mixed the two classes of trials with equal numbers in an experiment by labeling them. After preprocessing, the spatial features of recorded EEG signals were extracted for each paradigm by the common spatial patterns (CSP) method $[35,36]$. These features were then respectively classified by the support vector machine (SVM) classifier [37, 38]. We expected that our proposed paradigm could achieve significantly higher classification accuracies than the traditional paradigm so as to greatly improve the $\mathrm{BCI}$ performance.

\section{METHODS}

\section{A. Participants}

Eight healthy native Chinese speakers participated in this study. Their mean age is 22.6 years with standard deviation of 2.01. All the participants were recruited from Tongji University and with normal or corrected-to-normal vision. Written informed consent was obtained from all the participants. This study was approved by the Ethics Committee of the Medicine and Life Science Faculty at Tongji University. The experiment was conducted according to the approved guidelines.

\section{B. Convert Verb Reading Paradigm}

In this covert verb reading paradigm, each cue stimulus was a verb presented above an arrow pointing to the right or beside an arrow pointing to the downward (Fig. 1). Eighteen commonest verbs were used here, including nine referring to hand movements: “抬 (raise), 推 (push), 挂 (hang), 捞 (refloat), 拉 (pull), 挥 (wave), 抓 (grasp), 打 (hit), 摇 (shake)”; and nine referring to foot movements: “踩 (tramp), 跑 (run), 跳 (jump), 蹬 (pedal), 踹 (stamp), 踢 (kick), 蹦 (leap), 踏 (tread), 走 (walk)". All the verbs were selected through the questionnaire about the familiarity of the movements referred by the verbs.

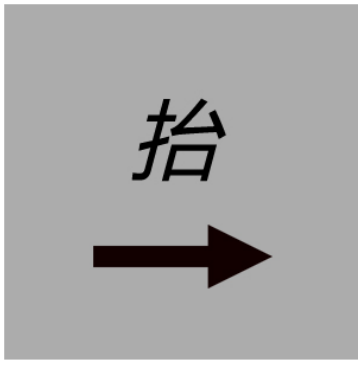

(A)

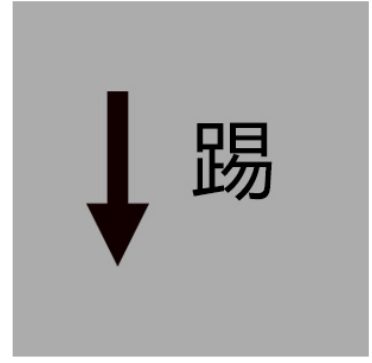

(B)
Fig. 1 Examples of proposed cue stimuli for covert verb reading during motor imagery of right hand movements (A) or foot movements (B).

There were two classes of trials in this paradigm. One class was for right hand motor imagery and the other was for foot motor imagery. Each trial began with a fixation cross presented in the center of the screen. This cross instructed participants to get ready for the experimental tasks. After 1 second, the fixation cross was replaced by a verb with an arrow, which appeared for 4 seconds. During this period, participants were instructed to covertly read the presented verb during motor imagery of the right hand or foot movement referred by the verb. Then, there was a blank screen for rest in a random duration between 1 second to 2 seconds. The timing of a trial in this paradigm was displayed in Fig. 2.

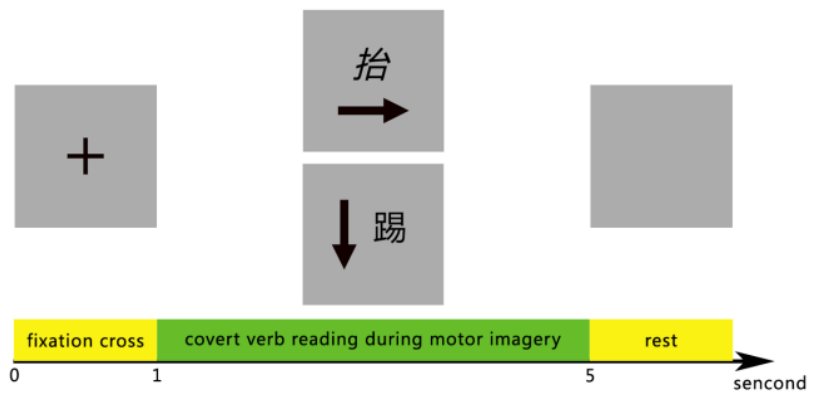

Fig. 2 Timing of a trial of the covert verb reading paradigm. Each trial consisted of ready, task and rest periods. Participants started to execute the experiment tasks while the verb with the arrow was presented in the screen.

\section{Design and Procedure}

There were 3 homogeneous runs. Each run involved 36 traditional and 36 covert verb reading trials. The trials for motor imagery of right hand and foot movements were equally distributed in each run. That is, there were equal traditional trials for motor imagery of right hand or foot movements, and equal new trials for covert reading of verbs referring to right hand or foot movements during imagining the corresponding movements. All the trials were presented in a pseudo random order. 
TABLE I

CLASSIFICATION ACCURACIES DURING MI AND VRMI CONDITIONS (IN\%)

\begin{tabular}{c|cccccccccc}
\hline Participant & S1 & S2 & S3 & S4 & S5 & S6 & S7 & S8 & $\begin{array}{c}\text { Mean } \\
\text { Accuracy }\end{array}$ \\
\hline MI & 57.96 & 66.85 & 60.19 & 66.67 & 96.30 & 69.44 & 70.56 & 61.11 & 68.63 \\
VRMI & 69.44 & 78.15 & 55.00 & 72.04 & 96.11 & 76.39 & 77.50 & 62.50 & 73.39 \\
\hline
\end{tabular}

Participants were seated in a comfortable chair in an acoustically and electrically shielded, dimly lit chamber. Their eyes were $80 \mathrm{~cm}$ away from the screen. In the traditional trials (MI condition), participants were asked to imagine right hand or foot movements cued by the presented arrows. In the covert verb reading trials (VRMI condition), they were instructed to read the presented verb covertly during imagining the movement referred by that verb. No feedback was provided throughout both paradigms. Participants were also required to keep all of their body parts as still as possible while they were performing the experimental tasks. After each run, there was a break for two minutes. When each participant finished the experiment, we verbally inquired of them about the task difficulty of the covert verb reading paradigm.

\section{Data acquisition and preprocessing}

EEG data were recorded from 64 electrodes using a 10-20 system Easycap (Brain Products). Eye blinks or movements were monitored by the electrodes on outer canthi and below each eye. There were another two electrodes used as reference by posited on the left and right mastoids. To ensure the effect of covert verb reading on motor imagery, electrode impedances below $50 \mathrm{k} \Omega$ were accepted since our experiment referred the EEG results reported by Dalla Volta et al. (2014). The signals were sampled at $500 \mathrm{~Hz}$ and filtered from 8 to $13 \mathrm{~Hz}$. This frequency band was with the best classification performance in our experiment and showed the largest difference between the covert verb reading paradigm and the traditional paradigm. After removing artefacts elicited by electrooculogram (EOG) and electromyogram (EMG), the continuous EEG signals were divided into epochs according to the labels.

\section{E. Data processing}

CSP is an effective method for feature extraction in BCI [35, 36]. We employed it in this experiment to extract features from the EEG signals.

Next, features extracted by CSP method were used to calculate a SVM classifier for parameter adjustment.

To appropriately estimate the classification accuracy, the data sets under the traditional and covert verb reading paradigms were divided into training and testing sets respectively. Each training set was used to calculate a SVM classifier, which is then used to classify the corresponding testing set. This procedure was repeated 15 times with different data parts into training and testing sets.

\section{RESULTS}

To evaluate the classification performance, we implemented a 5-fold cross-validation for each of the two paradigms. TABLE I showed the classification accuracies (in \%) for all the participants during MI and VRMI conditions and this result was also shown with the standard deviations in Fig. 3. Clearly, VRMI condition produced better classification accuracies for almost all the participants than MI condition. Since the classification accuracies for participant S3 and S5 were not increased by the covert verb reading paradigm, the data from the two participants was excluded from the following data analysis.

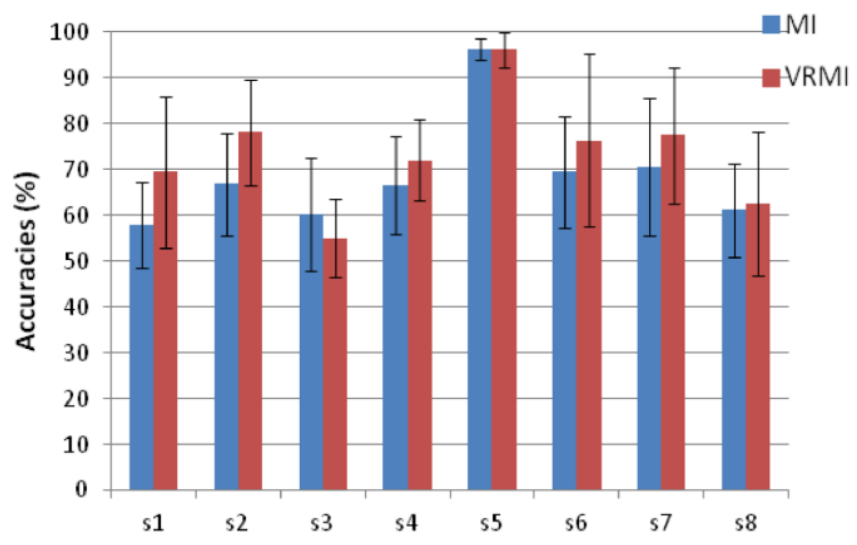

Fig. 3 Classification accuracies of all the participants from the traditional (MI) and covert verb reading (VRMI) paradigms.

Paired t test on the classification results showed that the classification accuracies were significantly increased in the VRMI condition ( $\mathrm{t}=4.661, \mathrm{P}=0.006$ ). For all the participants, we extracted the most important spatial patterns by CSP method over the parietal cortex which performs motor processing (Fig. 4). During MI condition, the motor imagery of right hand did not elicited reasonable ERD on the contralateral hemisphere. For most of the participants, the spatial patterns for the motor imagery of right hand were similar to that for the motor imagery of foot. By contrast, during the VRMI condition, most of right hand motor imagery elicited an increased EEG variance over the right hemisphere since the ERD of EEG took place on the left hemisphere. The pattern for motor imagery of right hand was mainly focused at electrode $\mathrm{C} 4$, which overlaid the motor hand area. However, the focus for foot imagery was at electrodes slightly right to $\mathrm{Cz}$. 


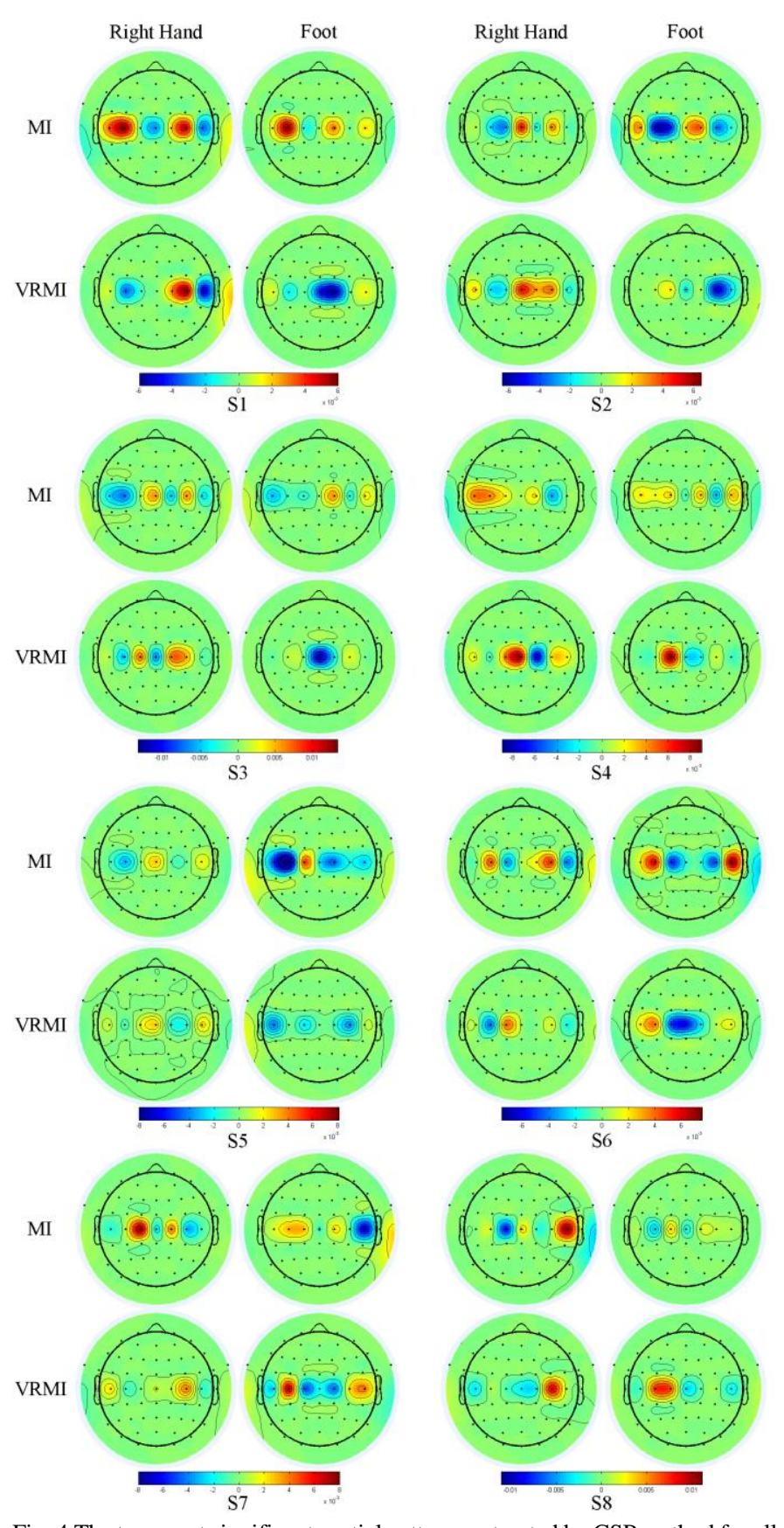

Fig. 4 The two most significant spatial patterns extracted by CSP method for all the participants, with data filtered by $8-13 \mathrm{~Hz}$.

To show the difference between the motor imagery of right hand and foot, grand-averaged power spectral densities (PSDs) were calculated for each condition (Fig. 5). For the covert verb reading paradigm, the energy from right hand motor imagery was higher than that from foot motor imagery at $\mathrm{C} 4$ and $\mathrm{Cz}$. For the traditional motor imagery paradigm, there was no significant feature between the two classes of motor imagery tasks at $\mathrm{C} 4$, while signals from right hand motor imagery were higher than that from foot motor imagery on most of the band at Cz.
$\mathrm{MI}$

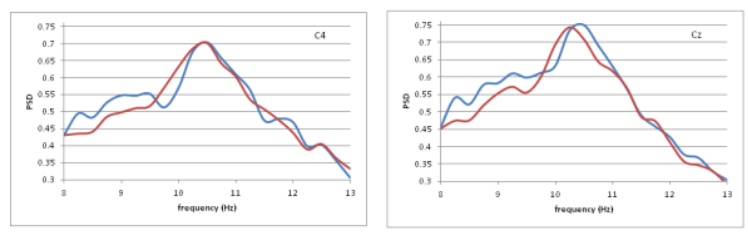

VRMI

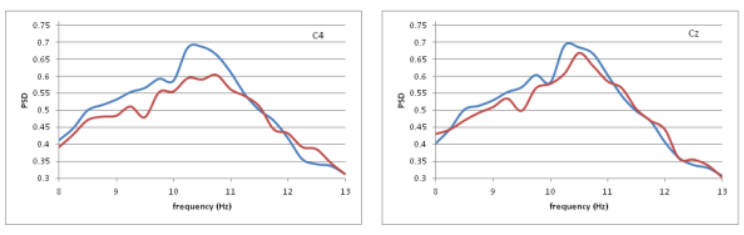

right hand foot

Fig. 5 The grand-averaged spectra of the two different paradigms at $\mathrm{C} 4$ and $\mathrm{Cz}$ (blue: right hand, red: foot).

\section{DISCUSSION}

The present work proposed a covert verb reading paradigm for motor imagery based BCI systems. Through the influence of verb processing on the brain activity of motor imagery, this new paradigm resulted in more easily discriminated features extracted from spatial patterns and significantly higher classification accuracies of EEG signals than the traditional paradigm. These results suggested that the covert verb reading paradigm could help users greatly improve their BCI performances.

Mu-rhythm changes on the basis of motor imagery over the motor cortex [39] and was reported to be task sensitive. In our experiment, the involvement of covert verb reading contributed to the reduced amplitude of mu rhythms for right hand motor imagery over the left hemisphere. This suggested that covert verb reading influenced brain activity of motor imagery and led to the clearer mu ERD over the corresponding brain regions. Embodied theories suggest that language understanding involves sensory and motor experiences related to the semantic meaning of that language [40-43]. Especially, the processing of verbs such as 'grasp' recruits the experiences related to the actions represented by those verbs [24, 44-47]. In previous studies, using the source estimation of multi-channel EEG, the processing of concrete verbs was found to activate the specific motor areas in a somatotopic manner [25]. Further, reading of the sentences including verbs related to hand actions was reported to elicit a greater mu ERD over the motor hand areas [48]. The present result that the ERD became clearer when covert verb reading was involved in the traditional paradigm of motor imagery in our experiment is consistent with these findings. Our results suggested that the involvement of covert verb reading is a promising approach to the improvement of operators' performance in the motor imagery based BCI systems.

On the basis of mu ERD, we decomposed the raw EEG signals into spatial patterns using CSP method. These patterns could maximize the difference between the EEG recordings during right hand and foot motor imagery. In VRMI condition, the clearer mu ERD resulted in more distinguishable spatial patterns than in traditional MI condition. These characteristic 
patterns defined the best directions that were most suitable for discrimination between the two classes of experimental tasks. Since the features used later for classification were calculated from the projections of EEG signals onto these best directions, the more distinguishable patterns resulted in the more easily classified features. Therefore, compared with the classification accuracies obtained from MI condition, the accuracies from VRMI condition were greatly improved.

In our results, the spatial patterns for motor imagery of foot in the VRMI condition were slightly right to that in the MI condition. This may be resulted from the influence of covert verb reading. Previous work reported that the reading of action words referring to hand or foot actions differentially activated motor areas that either were directly adjacent to or overlapped with the areas activated by actual movement of hands or feet [24]. According to this result, when motor imagery is accompanied with covert verb reading, the spatial patterns may appear a little deviation.

Although the average accuracy was greatly improved in the covert verb reading paradigm, there was one participant (S3) performing worse during this new paradigm than during the traditional paradigm, and another participant (S5) having almost same performances during the two paradigms. Our inquiry result showed that participants preferred to imagine different actions to verbs in their favor. The verbs used in the covert verb reading paradigm referred only some of their preferred actions. For some specific participants, the verbs which are not preferred by them may influence their performance of the motor imagery task. In our experiment, all other participants reported that there were no more than 3 verbs not referring to their preferred actions and they could quickly adapt to the covert reading and imagination tasks, whereas S3 was in trouble with 7 verbs when he imagined the actions. He reported that, compared with the traditional task, he was often in a muddle during the covert reading and imagination tasks. Therefore, the worse performance of S3 during the new paradigm might be derived from too many verbs in the experiment referring the actions that he did not prefer to imagine. These verbs puzzled him and made the new task more difficult for him. As for S5, he is a pro-operator and has been specifically trained for a long time under the traditional paradigm of motor imagery. He reported that not all of the verbs presented were used in his usual motor imagery, and there was a little maladjustment during the initial trials of the covert verb reading paradigm. This may be the reason that he could not perform better under the new paradigm. The results from S3 and S5 suggested that how to apply personalized verbs in the covert verb reading paradigm for more general usage may be worth investigating in our future studies.

\section{CONCLUSION}

In this work, we proposed a novel paradigm for motor imagery based BCI, in which the strategy of covert verb reading was utilized to enhance the traditional motor imagery. The significant improvement of $\mathrm{BCI}$ performance was achieved because of the enhanced mu ERD and more easily classified features from spatial patterns. The results from our experiment also provided evidence for the interaction between semantic processing and motor imagery, and suggested that semantic processing is helpful for the classification of motor imagery signals. This new paradigm can not only improve the traditional MI-BCI paradigm but also contribute to the training of novice users to adapt to a BCI system faster and more efficiently. Importantly, consistent with the traditional paradigm, this covert verb reading paradigm does not need any body actions. It is therefore appropriate for patients with motor or limb disability, and can help them more easily communicate with external environment. In the future studies, personalized verb selection for covert verb reading will be an interesting issue for the further investigation. Moreover, how to use the multi-class of imagined actions in this new paradigm and how to accurately translate the corresponding EEG signals to the multi-class of control signals for devices will also be worth studying.

\section{REFERENCES}

[1]M. Jeannerod, "The representing brain: Neural correlates of motor intention and imagery," Behav. Brain Sci., vol. 17, no. 2, pp. 187-202, Jun. 1994.

[2]M. Steriade and R. R. Llinás, "The functional states of the thalamus and the associated neuronal interplay," Physiol. Rev., vol. 68, no. 3, pp. 649-742, Jul. 1988.

[3] G. Pfurtscheller and A. Berghold, "Patterns of cortical activation during planning of voluntary movement," Electroenceph. Clin. Neurophysiol., vol. 72, no.3, pp. 250-258, Mar. 1989.

[4] G. Pfurtscheller, C. Neuper, D. Flotzinger, and M. Pregenzer, "EEG-based discrimination between imagination of right and left hand movement,"

Electroenceph. Clin. Neurophysiol., vol. 103, no.6, pp. 642-651, Dec. 1997.

[5]D. J. Mcfarland, L. A. Miner, T. M. Vaughan, and J. R. Wolpaw, "Mu and Beta Rhythm topographies during motor imagery and actual movements,"

Brain Topogr., vol. 12, no. 3, pp. 177-186, Mar. 2000.

[6]J. R. Wolpaw, N. Birbaumer, D. J. McFarland, G. Pfurtscheller, and T. M. Vaughan, "Brain-computer interfaces for communication and control," Clin. Neurophysiol., vol. 113, no.6, pp. 767-791, Jun. 2002.

[7]G. Pfurtscheller and F. H. L. D. Silva, "Event-related EEG/MEG synchronization and desynchronization: Basic principles," Clin. Neurophysiol., vol. 110, no. 11, pp. 1842-1857, Nov. 1999.

[8] G. Pfurtscheller, C. Brunner, A. Schlögl, and F. H. L. D. Silva, "Mu rhythm (de)synchronization and EEG single-trial classification of different motor imagery tasks," Neuroimage, vol. 31, no. 1, pp. 153-159, May 2006.

[9]G. E. Fabiani, D. J. Mcfarland, J. R. Wolpaw, and G. Pfurtscheller,

"Conversion of EEG activity into cursor movement by a brain-computer interface (BCI)," IEEE T. Neur. Sys. Reh., vol. 12, no.3, pp. 331-338, Sep. 2004.

[10] D. J. Mcfarland, W. A. Sarnacki and J. R. Wolpaw,

"Electroencephalographic (EEG) control of three-dimensional movement," $J$. Neural. Eng., vol. 7, no. 3, pp. 189-190, Jun. 2010.

[11] A. Nijholt, P. O. Bos and B. Reuderink, "Turning shortcomings into challenges: Brain-computer interfaces for games," Entertain. Comput., vol. 1, no. 2, pp. 85-94, Apr. 2009

[12] Q. B. Zhao, L. Q. Zhang and A. Cichocki, "EEG-based asynchronous BCI control of a car in 3D virtual reality environments," Sci. Bull., vol. 54, no. 1, pp. 78-87, Jan. 2009

[13] A. Fattouh, O. Horn and G. Bourhis, "Emotional BCI control of a smart wheelchair," Int. J. Comput. Sci., vol. 10, no. 1, pp. 32-36, May. 2013.

[14] Siuly, Y. Li and P. Wen, "Modified CC-LR algorithm with three diverse feature sets for motor imagery tasks classification in EEG based

brain-computer interface," Comput. Meth. Prog. Bio., vol. 113, no. 3, pp. 767-780, Mar. 2014. 
[15] Kołodziej M, Majkowski A, Rak R J. "A new method of EEG classification for $\mathrm{BCI}$ with feature extraction based on higher order statistics of wavelet components and selection with genetic algorithms," International Conference on Adaptive and Natural Computing Algorithms. Springer Berlin Heidelberg, pp. 280-289, 2011.

[16] J. Kevric and A. Subasi, "The impact of mspca signal de-noising in real-time wireless brain computer interface system," Southeast Eur. J. Soft Comput., vol. 4, no. 1, pp. 43-47, Mar. 2015

[17] B. Blankertz, G. Dornhege, M. Krauledat, K. Müller, and G. Curio, "The non-invasive Berlin brain-computer interface: fast acquisition of effective performance in untrained subjects," NeuroImage, vol. 37, no.2, pp. 539-550, Aug. 2007.

[18] C. Neuper, R. Scherer, S. Wriessnegger, and G. Pfurtscheller, "Motor imagery and action observation: modulation of sensorimotor brain rhythms during mental control of a brain-computer interface," Clin. Neurophysiol., vol. 120, no. 2, pp. 239-247, Feb. 2009.

[19] J. Yue, Z. Zhou, J. Jiang, Y. Liu, and D. Hu, "Balancing a simulated inverted pendulum through motor imagery: an EEG-based real-time control paradigm," Neurosci. Lett., vol. 524, no. 2, pp. 95-100, Aug. 2012.

[20] T. Kondo, M. Saeki, Y. Hayashi, K. Nakayashiki, and Y. Takata, "Effect of instructive visual stimuli on neurofeedback training for motor imagery-based brain-computer interface," Hum. Movement Sci., vol. 43, pp. 239-249, Oct. 2015.

[21] K. A. McCreadie, D. H. Coyle and G. Prasad, "Is sensorimotor BCI performance influenced differently by mono, stereo, or 3-D auditory

feedback?" IEEE T. NEUR. SYS. REH., vol. 22, no. 3, pp. 431-440, May 2014. [22] T. Yu, J. Xiao, F. Wang, R. Zhang, Z. Gu, A. Cichocki, and Y. Li, "Enhanced motor imagery training using a hybrid BCI with feedback," IEEE T. Bio-Med Eng., vol. 62, no.7, pp. 1706-1717, Jul. 2015.

[23] J. Li and L. Zhang, "Active training paradigm for motor imagery BCI," Exp. Brain Res., vol. 219, no. 2, pp. 245-254, Mar. 2012.

[24] O. Hauk, I. Johnsrude and F. Pulvermüller, "Somatotopic representation of action words in human motor and premotor cortex," Neuron, vol. 41, no. 2, pp. 301-307, Jan. 2004.

[25] R. Dalla Volta, M. Fabbri-Destro, M. Gentilucci, and P. Avanzini,

"Spatiotemporal dynamics during processing of abstract and concrete verbs: An ERP study," Neuropsychologia, vol. 61, no. 1, pp. 163-174, Aug. 2014.

[26] M. Oliveri, C. Finocchiaro, K. Shapiro, and M. Gangitano, "All talk and no action: a transcranial magnetic stimulation study of motor cortex activation during action word production," J. Cognitive Neurosci., vol. 16, no. 3, pp. 374-381, Apr. 2004.

[27] V. Boulenger, A. C. Roy, Y. Paulignan, V. Deprez, M. Jeannerod, and T. A. Nazir, "Cross-talk between language processes and overt motor behavior in the first 200 msec of processing," J. Cognitive Neurosci., vol. 18, no. 10, pp. 1607-1615, Oct. 2006.

[28] V. Boulenger, B. Y. Silber, A. C. Roy, Y. Paulignan, M. Jeannerod, and T. A. Nazir, "Subliminal display of action words interferes with motor planning: A combined EEG and kinematic study," J. Physiol-Paris., vol. 102, pp. 130-136, May 2008.

[29] T. A. Nazir, V. Boulenger, A. Roy, B. Silber, M. Jeannerod, and Y. Paulignan, "Language-induced motor perturbations during the execution of a reaching movement," Q. J. Exp. Psychol., vol. 61, no. 6, pp. 933-943, Jan. 2008.

[30] A. D. Rodriguez, M. L. McCabe, J. R. Nocera, and J. Reilly, "Concurrent word generation and motor performance: further evidence for language-motor interaction," Plos One, vol. 7, no. 5, e37094, May 2012.

[31] L. Zhang, M. Wininger and D. A. Rosenbaum, "Word generation affects continuous hand movements," J. Motor Behav., vol. 46, no. 2, pp. 115-123, Feb. 2014.

[32] G. Pfurtscheller and C. Neuper, "Motor imagery activates primary sensorimotor area in humans," Neurosci. Lett., vol. 239, no. 239, pp. 65-68, Dec. 1997.

[33] C. A. Porro, M. P. Francescato, V. Cettolo, M. E. Diamond, P. Baraldi, C. Zuiani, M. Bazzocchi, and P. P. Di, "Primary motor and sensory cortex activation during motor performance and motor imagery: a functional magnetic resonance imaging study," J. Neurosci., vol. 16, no. 23, pp. 7688-7698, Dec. 1996.

[34] C. Stippich, H. Ochmann and K. Sartor, "Somatotopic mapping of the human primary sensorimotor cortex during motor imagery and motor execution by functional magnetic resonance imaging," Neurosci. Lett., vol. 331, no. 1, pp. 50-54, Oct. 2002.

[35] J. Müllergerking, G. Pfurtscheller and H. Flyvbjerg, "Designing optimal spatial filters for single-trial EEG classification in a movement task," Clin.

Neurophysiol., vol. 110, no. 5, pp. 787-798, May 1999.

[36] H. Ramoser, J. Muller-Gerking and G. Pfurtscheller, "Optimal spatial filtering of single trial EEG during imagined hand movement," IEEE Trans.

Rehabil. Eng., vol. 8, no. 4, pp. 441-446, Dec. 2000.

[37] V. Vapnik, "The nature of statistical learning theory," New York, Springer, 2000, pp. 988 - 999.

[38] M. A. Hearst, S. T. Dumais, E. Osuna, J. Platt, and B. Scholkopf, "Support vector machines," IEEE Intell. Syst., vol. 13, no. 4, pp. 18-28, Aug. 1998.

[39] C. Llanos, M. Rodriguez, C. Rodriguez-Sabate, I. Morales, and M. Sabate, "Mu-rhythm changes during the planning of motor and motor imagery actions," Neuropsychologia, vol. 51, no. 6, pp. 1019-1026, May 2013.

[40] M. H. Fischer and R. A. Zwaan, "Embodied language: a review of the role of the motor system in language comprehension," Q. J. Exp. Psychol., vol. 61, no. 6, pp. 825-850, May 2008.

[41] V. Gallese and G. Lakoff, "The Brain's concepts: the role of the Sensory-motor system in conceptual knowledge," Cogn. Neuropsychol., vol. 22, no. 3, pp. 455-479, May 2005.

[42] F. Pulvermüller, O. Hauk, V. V. Nikulin, and R. J. Ilmoniemi,

"Functional links between motor and language systems," Eur. J. Neurosci., vol. 21, no. 3, pp. 793-797, Feb. 2005.

[43] R. A. Zwaan and L. J. Taylor, "Seeing, acting, understanding: Motor resonance in language comprehension," J. Exp. Psychol. Gen., vol. 135, no. 1, pp. 1-11, Feb. 2006.

[44] O. Hauk and F. Pulvermüller, "Neurophysiological distinction of action words in the fronto-central cortex," Hum. Brain mapp., vol. 21, no. 3, pp. 191-201, Mar. 2004.

[45] V. D. Lafuente and R. Romo, "Language Abilities of Motor Cortex," Neuron, vol. 41, no. 2, pp. 178-180, Jan. 2004.

[46] F. Pulvermuller, "Brain mechanisms linking language and action," Nat. Rev. Neurosci., vol. 6, no. 7, pp. 576-582, Jul. 2005.

[47] F. Pulvermüller and L. Fadiga, "Active perception: sensorimotor circuits as a cortical basis for language," Nat. Rev. Neurosci., vol. 11, no. 5, pp. 351-360, May 2010.

[48] F. Alemanno, E. Houdayer and M. Cursi, "Action-related semantic content and negation polarity modulate motor areas during sentence reading: an event-related desynchronization study," Brain Res., vol. 1484, no. 16, pp. 39-49, Nov. 2012. 